\title{
INTEGRAL BACKSTEPPING SLIDING MODE Control Of Chaotic Forced Van Der Pol OSCILLATOR
}

\author{
Ismaila Adeniyi Kamil and Samuel Oyelekan Oladipo \\ Department of Electrical \& Electronic Engineering,University of Ibadan, Nigeria
}

\begin{abstract}
Forced Van der Pol oscillator exhibits chaotic behaviour and instability under certain parameters and this poses a great threat to the systems where it has been applied hence, the need to develop a control method to stabilize and control chaos in a Forced Van der Pol oscillator so as to avoid damage in the controlled system and also to prevent unmodeled dynamics from being introduced in the system. Sliding Mode control makes use of the regulatory variables derived from the controlled Lyapunov function to bring the new variables to stability. The essence of using Integral Backstepping was to prevent chattering which can occur in the control input and can cause instability to the system by igniting unmodeled dynamics. Simulation was done using MATLAB and the results were provided to show the effectiveness of the proposed control method. Integral Backstepping Sliding Mode control method was effective towards stability and chaos control. It was also robust towards matched and unmatched disturbance.
\end{abstract}

\section{KEYWORDS}

Chaos Control, Lyapunov Function, Van der Pol Oscillator, Sliding Mode, Integral Backstepping

\section{INTRODUCTION}

The Van der Pol Oscillator is the keystone for studying systems with limit cycle oscillations due to its unique nature. It can display oscillations of fixed amplitude and fixed period without external excitation. These oscillations are called limit cycles, or self-excited oscillations [1], [2], [3].

Also, it has been observed that Van der Pol oscillator can exhibit chaotic behaviour depending on the values of its parameters and initial conditions. For stable linear systems, small differences in initial conditions can only cause small differences in output. Nonlinear systems however, can display a phenomenon called chaos, whereby the system output is extremely sensitive to initial conditions. The essential feature of chaos is the unpredictability of the system output [4].

In many applications, chaos has been viewed as an undesirable phenomenon which may damage such physical systems, especially in mechanical non-linear devices such as coupled oscillators [5]. The first control strategy was suggested by [6] in order to stabilize the unstable periodic orbits. Different methods have since been developed for controlling chaotic systems [7]. [8] proposed an adaptive controller to control chaos in Lorenz system. [9] developed a neural networks-based control law for chaotic systems. However, many of these proposed methods are based on supposed knowledge of all the state variables which cannot always be measured due to noise effect on sensors. Consequently, the design of a state-observer is needed to estimate the unmeasured velocity signals of such a system in order to construct an adequate control law. In literature, several types of observers have been proposed for chaotic systems [10]. One of such is an observer-based backstepping control scheme to stabilize a class of chaotic systems proposed in 
International Journal of Control Theory and Computer Modeling (IJCTCM) Vol.8, No.1/2/3/4, October 2018

[11]. These approaches seem to give good results on controlling chaos, however, many of them failed for dynamical systems in the presence of external forces (perturbing terms).

In this research work, Sliding Mode control based on Integral Backstepping control technique was used to control a chaotic forced Van der Pol Oscillator

\section{Materials AND MethodS}

This section presents the structure of research methodology employed in order to realize the research aim and objectives. The Van der Pol system was modelled containing the states of the system, the damping function, the actuator and the forcing function as shown in equation 1 . The dynamic of the system was altered during the integral backstepping algorithm and sliding mode control algorithm. A new control law was derived and was passed to the system through the actuator.

\subsection{Integral Backstepping Control for Forced Van der Pol Oscillator}

In the integral backstepping control procedure, the system equation was treated as series of two systems connected together, each with a single input and output. A change of coordinate was introduced during the recursive design process and a new state vector was formed. For the first system the state was chosen as a virtual control input. Controlled Lyapunov functions were used in order to stabilize the system.

Considering the states of the system $\ddot{x}, \dot{x}$ and $x$, the damping function $\sigma$ and the forcing function $P \cos \alpha t$, the equation of a forced Van der Pol Oscillator is generated as shown in equation 1

$$
\ddot{x}+\sigma\left(x^{2}-1\right) \dot{x}+x=u+P \cos \alpha t
$$

where $\sigma, \alpha$ and $P$ are known constant parameters and $u$ is the actuator. The system can be viewed as a cascade of two systems as shown below,

$$
\begin{aligned}
& \dot{m}=g(m)+f \\
& \dot{f}=u+P \cos \beta t
\end{aligned}
$$

where $m$ and $f$ are the system states, $u$ is the control input and $g(x)$ is the known function. In the integrator backstepping case, $f$ is used as an input for the stabilization of $m$ and can therefore be referred to as the virtual control.

The first backstepping variable is chosen as

$$
z_{1}=m
$$

The virtual control is defined as

$$
f=\alpha+z_{2}
$$

where $\alpha$ is the stabilizing function and $z_{2}$ is the new state variable.

Therefore,

$$
\dot{z}_{1}=g(m)+f
$$

Substituting (5) into (6), the equation becomes

$$
\dot{z}_{1}=g(m)+\alpha+z_{2}
$$


It should be noted that the new state variable $z_{2}$ will not be used in the first step but its presence is needed to couple $z_{1}$ system to the next system.

Integrator backstepping implies that the coordinates during the recursive design are changed from

$$
x=\left[\begin{array}{ll}
m & f
\end{array}\right]^{\mathrm{T}} \text { to } z=\left[z_{1} z_{2}\right]^{\mathrm{T}}
$$

A controlled Lyapunov function (CLF) is given as

$$
V_{1}=\frac{1}{2} z_{1}^{2}
$$

Hence,

$$
\dot{V}_{1}=z_{1} \dot{z}_{1}
$$

Therefore from (6) we have

$$
\dot{V}_{1}=z_{1}[g(m)+\alpha]+z_{1} z_{2}
$$

The stabilizing function $\alpha$, is chosen as a feedback linearizing controller. Therefore from (5) we have,

$$
\alpha=f-z_{2}
$$

and

$$
\dot{V}_{1}=z_{1}[g(m)+\alpha]=g(m) z_{1}+\alpha z_{1}
$$

If $\dot{V} \leq-k_{1} z_{1}^{2}$ where $k$ is a positive gain then,

$$
\alpha=-k_{1} z_{1}-g(m)
$$

Substituting (10) into (9) gives

$$
\begin{aligned}
& \dot{V}_{1}=z_{1}\left[g(m)-k_{1} z_{1}-g(m)\right]+z_{1} z_{2} \\
& \dot{V}_{1}=-k_{1} z_{1}{ }^{2}+z_{1} z_{2}
\end{aligned}
$$

By making use of (8), we have

$$
\dot{z}_{1}=-k_{1} z_{1}+z_{2}
$$

If $z_{2}=0$ then $z_{1}$ is stabilized. Hence, we focus on $z_{2}$ which is computed by differentiating (5) giving

$$
\dot{f}=\dot{\alpha}+\dot{z}_{2}
$$

Recall that $\dot{f}=u+P \cos \beta t$ therefore,

$$
\dot{z}_{2}=u+P \cos \beta t-\dot{\alpha}
$$

The CLF for $z_{2}$ is given as,

$$
\begin{aligned}
& V_{2}=V_{1}+\frac{1}{2} z_{2}{ }^{2} \\
& \dot{V}_{2}=\dot{V}_{1}+z_{2} \dot{z}_{2}
\end{aligned}
$$


International Journal of Control Theory and Computer Modeling (IJCTCM) Vol.8, No.1/2/3/4, October 2018

Therefore,

$$
\begin{aligned}
& \dot{V}_{2}=-k_{1} z_{1}{ }^{2}+z_{1} z_{2}+z_{2} \dot{z}_{2} \\
& \dot{V}_{2}=-k_{1} z_{1}{ }^{2}+z_{1} z_{2}+z_{2}(u+P \cos \beta t-\dot{\alpha})
\end{aligned}
$$

It will be observed that $u$ appears on the second part of the equation, therefore the choice of $u$ is taken as

$$
\dot{V}_{2}=z_{1} z_{2}+z_{2} u+z_{2} P \cos \beta t-z_{2} \dot{\alpha}
$$

Let $\dot{V} \leq-k_{2} z_{2}{ }^{2}$

$$
u=-k_{2} z_{2}-z_{1}-P \cos \beta t+\dot{\alpha}
$$

Substituting 17 into 16 gives

$$
\dot{V}_{2}=-k_{1} z_{1}{ }^{2}-k_{2} z_{2}{ }^{2}
$$

To implement the control law expressions terms involving time derivatives of the states should be avoided. Hence, $\dot{\alpha}$ will be evaluated in (17) without using the state derivatives, by differentiating it along the trajectory of $m$.

From (10),

Therefore,

$$
\alpha=-k_{1} z_{1}-g(m)
$$

$$
\begin{aligned}
\dot{\alpha}= & -\frac{\partial g(m)}{\partial x} \dot{m}-k_{1} \dot{m} \\
\dot{\alpha}= & {\left[-\frac{\partial g(m)}{\partial x}-k_{1}\right] \dot{m} } \\
& \dot{\alpha}=-\left[\frac{\partial g(m)}{\partial x}+k_{1}\right] g(m)+f
\end{aligned}
$$

Hence the final expression for the control law $u$ is given as,

$$
u=-\left[\frac{\partial g(m)}{\partial x}+k_{1}\right](g(m)+f)-k_{2} z_{2}-z_{1}-P \cos \beta t
$$

Substituting for $z_{2}$ from (12) into (20), $u$ becomes

$$
u=-\left[\frac{\partial g(m)}{\partial x}+k_{1}\right](g(m)+f)-k_{2}\left(\dot{z}_{1}+k_{1} z_{1}\right)-z_{1}-P \cos \beta t
$$

Recall that $\dot{z}_{1}=\dot{m}$, therefore,

$$
u=-\left[\frac{\partial g(m)}{\partial x}+k_{1}\right](g(m)+f)-k_{2}\left(g(m)+f+k_{1} z_{1}\right)-z_{1}-P \cos \beta t
$$

And finally

$$
\begin{gathered}
u=-\left[\frac{\partial g(m)}{\partial x}+k_{1}\right](g(m)+f)-k_{2}\left(g(m)+f+k_{1} z_{1}\right)-z_{1}-P \cos \beta t \\
u=-\left[\frac{\partial g(m)}{\partial x}+k_{1}\right]\left(-\sigma\left(x^{2}-1\right) \dot{x}-x\right)-k_{2}\left(-\sigma\left(x^{2}-1\right) \dot{x}-x+k_{1} z_{1}\right)-z_{1}-P \cos \beta t
\end{gathered}
$$

where $\dot{x}$ and $x$ are the states of the system, $\sigma$ is the damping function, $k_{1}$ and $k_{2}$ are the positive gain, $z_{1}$ is the backstepping parameter, $P \cos \beta t$ is the forcing function and $g(m)$ is the known Van der Pol oscillator function. 


\subsection{Sliding Mode Control Algorithm}

In order to design the sliding surface, the system equations obtained in terms of the transferred coordinates are considered from equations (12) and (13)

$$
\begin{aligned}
& \dot{z}_{1}=-k_{1} z_{1}+z_{2} \\
& \dot{z}_{2}=u+P \cos \beta t-\dot{\alpha}
\end{aligned}
$$

Conventional linear sliding surface design $\mathrm{s}$ is defined as

$$
s=\gamma z
$$

where $\gamma$ is the sliding surface parameter which depends upon the system equations.

$$
\begin{aligned}
& \gamma=\left[\begin{array}{ll}
\gamma_{1} & 1
\end{array}\right] \text { and } z=\left[z_{1} z_{2}\right]^{\mathrm{T}} \\
& s=\gamma_{1} z_{1}+z_{2} \\
& \dot{s}=\gamma_{1} \dot{z}_{1}+\dot{z}_{2}
\end{aligned}
$$

Substituting (12) and (13) into (22) gives

$$
\dot{s}=-\gamma_{1} k_{1} z_{1}+\gamma_{1} z_{2}+u+P \cos \beta t-\dot{\alpha}
$$

The new CLF is obtained containing the sliding surface $s$

$$
\begin{aligned}
& V_{3}=\frac{1}{2}\left(z_{1}{ }^{2}+z_{2}{ }^{2}+s^{2}\right) \\
& \dot{V}_{3}=\dot{V}_{1}+\dot{V}_{2}+s \dot{s}
\end{aligned}
$$

Substituting for $\dot{V}_{1}, \dot{V}_{2}$ and $\dot{S}$ from (11), (16) and (23)

$$
\dot{V}_{3}=-k_{1} z_{1}{ }^{2}-k_{2} z_{2}{ }^{2}+s\left(-\gamma_{1} k_{1} z_{1}+\gamma_{1} z_{2}+u+P \cos \beta t-\dot{\alpha}\right)
$$

In order to force the system to stay on the sliding mode $u$ is chosen such that $\dot{s}=0$ Applying the sliding control

$$
u_{f}=u-\operatorname{sgn}(s)
$$

where $u_{f}$ is the final control signal and $s g n$ is the sign function

$$
\operatorname{sgn}= \begin{cases}+1 & \text { if } s>0 \\ 0 & \text { if } s=0 \\ -1 & \text { if } s<0\end{cases}
$$

Therefore

$$
u_{f}=\gamma_{1} k_{1} z_{1}-\gamma_{1} z_{2}-P \cos \beta t-\dot{\alpha}-\operatorname{sgn}(s)
$$

Substitute for $\dot{\alpha}$

$$
u_{f}=\gamma_{1} k_{1} z_{1}-\gamma_{1} z_{2}-P \cos \beta t+\left[\frac{\partial g(m)}{\partial x}+k_{1}\right] g(m)+f-\operatorname{sgn}(s)
$$


International Journal of Control Theory and Computer Modeling (IJCTCM) Vol.8, No.1/2/3/4, October 2018

$u_{f}=\gamma_{1} k_{1} z_{1}-\gamma_{1} z_{2}-P \cos \beta t+\left[\frac{\partial g(m)}{\partial x}+k_{1}\right]\left(-\sigma\left(x^{2}-1\right) \dot{x}-x\right)-\operatorname{sgn}(s)(25)$

Where $\dot{x}$ and $x$ are the states of the system, $\sigma$ is the damping function, $\gamma_{1}$ is the sliding surface parameter, $k_{1}$ is the positive gain, $z_{1}$ is the backstepping parameter, $z_{2}$ is the new state variable, $P \cos \beta t$ is the forcing function, $g(m)$ is the known Van der Pol oscillator function, $s$ is the sliding surface and $s g n$ is the sign function.

\subsection{System Simulation}

Stability and chaos control in an oscillator are very important in order to get the most effective behaviour and expected dynamics in its application. The more the stability the more the pleasantness in behaviour of the system.The system parameters are $c$ which is the damping function, $x_{0}$ and $v_{0}$, the initial conditions, $p$ the amplitude and $\omega$ the angular frequency.

The unforced state of the Van der Pol Oscillator (VPO) system was first simulated to investigate the behaviour of the system with parameter valuesc $=2, x_{0}=-2, v_{0}=2, p=100$ and $\omega=7$ in the absence of a control signal. The behaviours with the addition of integral backstepping control (IBC) and integral backstepping sliding mode control were then investigated through simulation.The process was repeated with the inclusion of a matched disturbance of $2 \sin (3 \mathrm{t})$. Also, the total harmonic distortion (THD) was evaluated for all cases.

\section{RESULTS AND DISCUSSION}

The result of the effects of the Integral Backstepping control and the Integral Backstepping Sliding Mode control method developed and simulated were illustrated and discussed in this section.

Figures 1(a) - (c) show that the forced Van der Pol oscillator is chaotic with parameter values $c=2, x_{0}=-2, v_{0}=2, p=100$ and $\omega=7$ in the absence of an applied control signal. The resulting signal oscillates beyond the evolving values thereby demonstrating a chaotic behaviour and instability even without a matched disturbance being added, with a THD of $-27.50 \mathrm{~dB}$ giving a distortion factor of $4.2 \%$.

Figures 2(a)-(c) show that the Forced Van der Pol oscillator with matched disturbance added is more chaotic and displays a THD of $-29.24 \mathrm{~dB}$ and a distortion factor of $5 \%$.

Figures 3(a)-(c) shows the performance of integral backstepping control method on a chaotic forced Van der Pol oscillator. The resulting signal shows a little deviation from the evolving value at position $\mathrm{x}(\mathrm{t})=-2.44$ and $\mathrm{x}(\mathrm{t})=1.57$ and $\mathrm{t}=0.25 \mathrm{sec}$ and $\mathrm{t}=0.88 \mathrm{sec}$ and gradually approaches stable oscillation between evolving values. The result of the simulation implies that the effect of chaos has been greatly reduced as shown in the phase portrait when compared to forced Van der Pol oscillator without an applied control signal. The system's response however displays a THD value of $-28.46 \mathrm{~dB}$ with a distortion factor of $8 \%$.

Figures 4(a)-(c) shows the performance of integral backstepping control method on a chaotic forced Van der Pol oscillator with matched disturbance. The resulting signal shows a little deviation from the evolving value at position $\mathrm{x}(\mathrm{t})=-2.434$ and $\mathrm{x}(\mathrm{t})=1.782$ and $\mathrm{t}=0.244 \mathrm{sec}$ and $\mathrm{t}=0.868 \mathrm{sec}$. It gradually approaches stable oscillation between evolving values. The result of the simulation implies that the control method is little less effective on chaos control as can be seen in Figure 4(a). 
International Journal of Control Theory and Computer Modeling (IJCTCM) Vol.8, No.1/2/3/4, October 2018

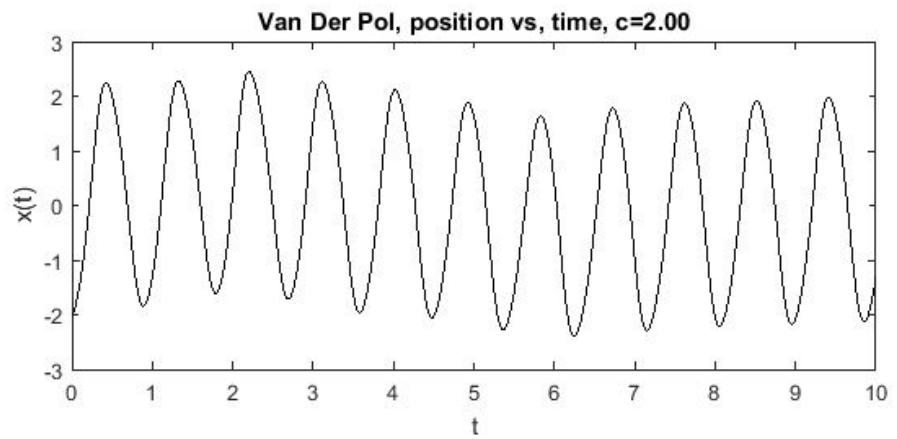

(a)

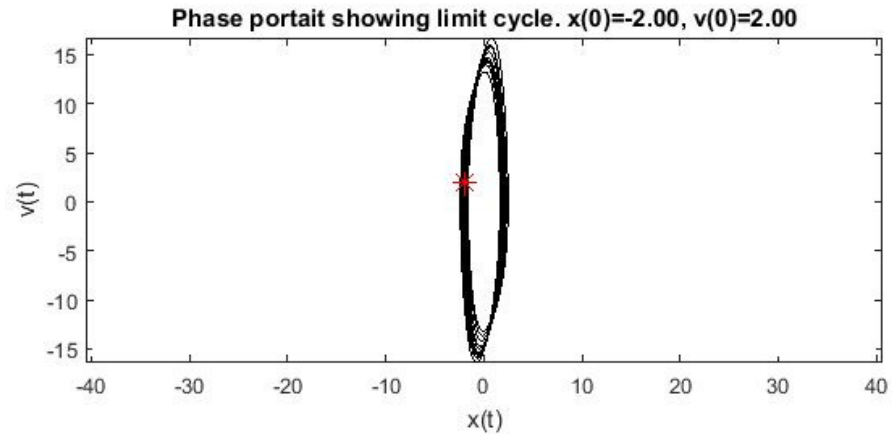

(b)

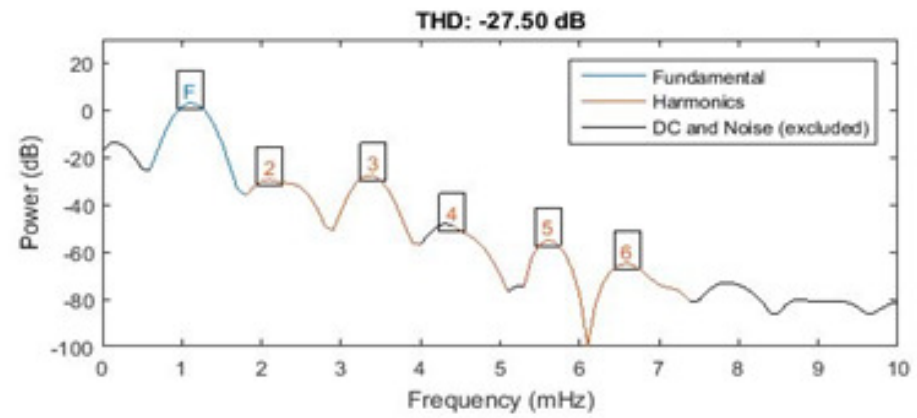

(c)

Figure 1. Chaotic Forced Van der Pol Oscillator (a) Time series (b) Phase portrait (c)Total Harmonic Distortion diagram 
International Journal of Control Theory and Computer Modeling (IJCTCM) Vol.8, No.1/2/3/4, October 2018

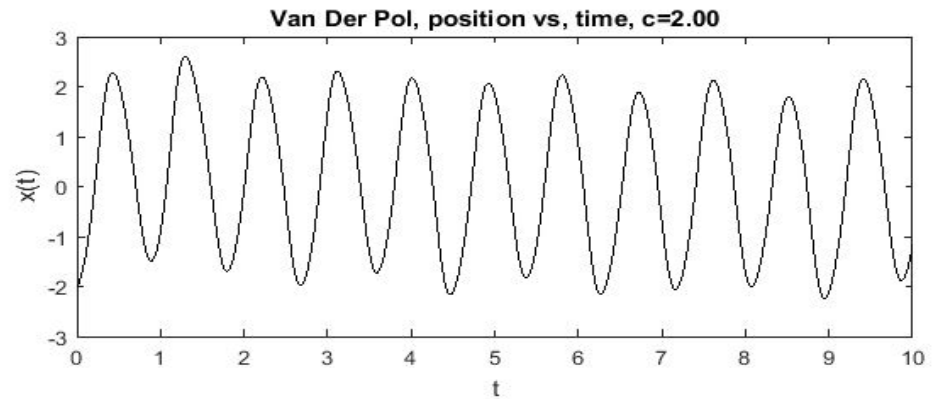

(a)

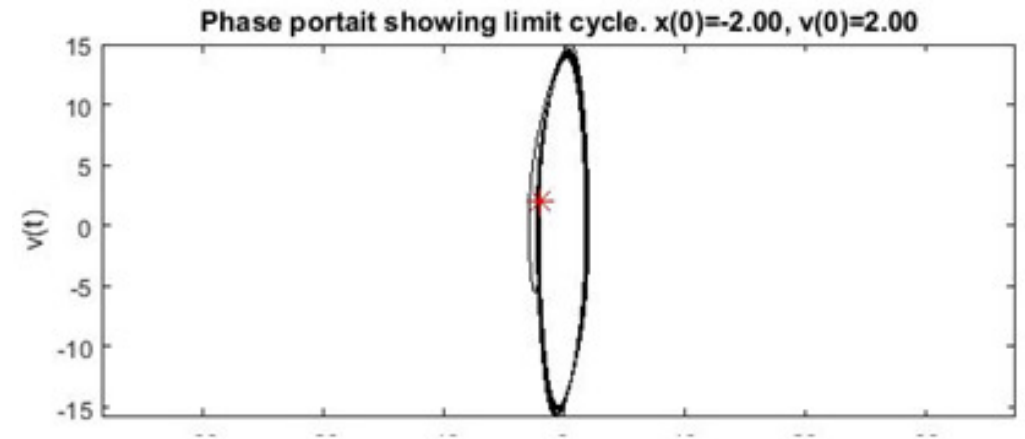

(b)

THD: $-28.46 \mathrm{~dB}$

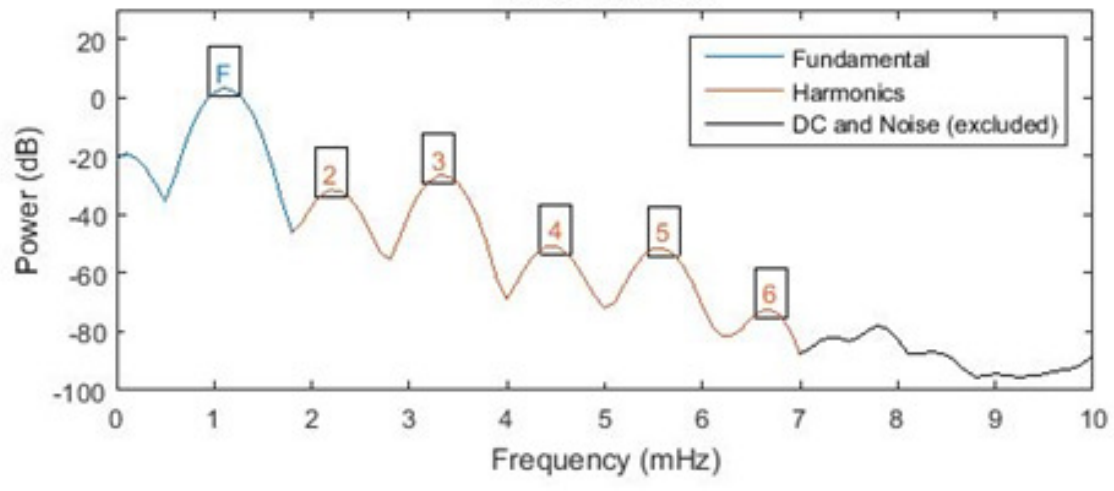

(c)

Figure 2. Chaotic Forced Van der Pol Oscillator with matched disturbance (a) Time series (b) Phase portrait (c)Total Harmonic Distortion diagram 
International Journal of Control Theory and Computer Modeling (IJCTCM) Vol.8, No.1/2/3/4, October 2018

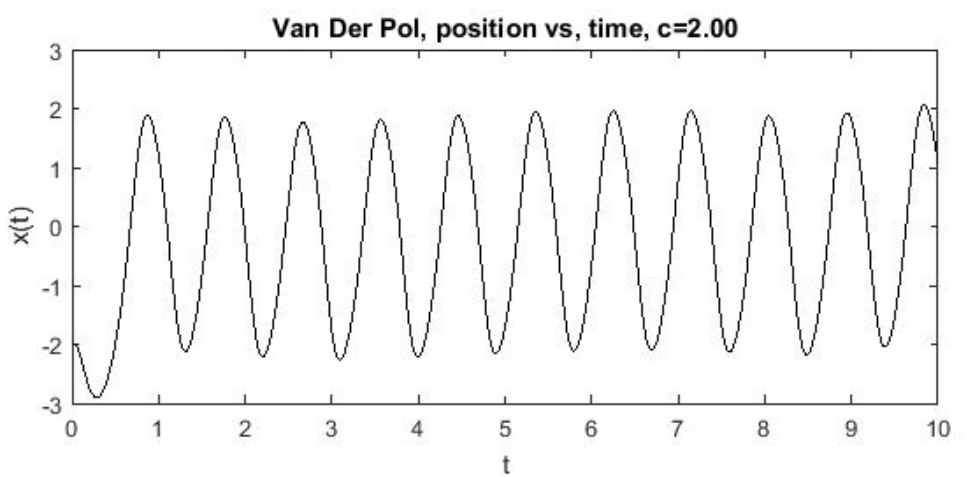

(a)

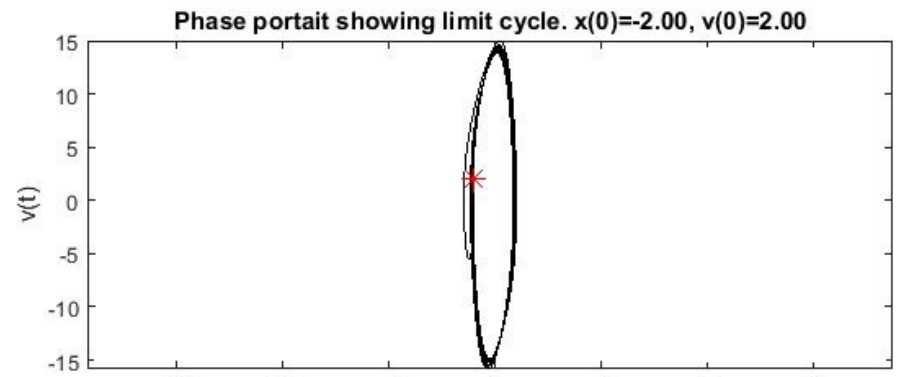

(b)

THD: $-\mathbf{2 8 . 4 6 \mathrm { dB }}$

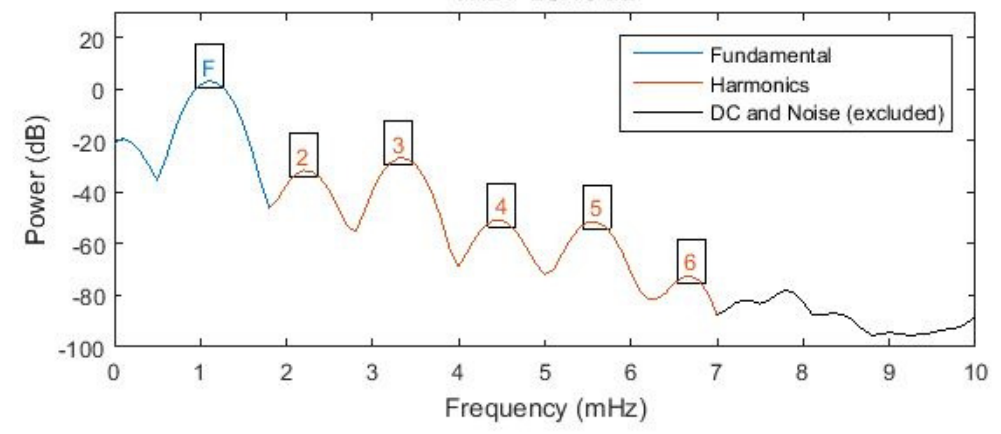

(c)

Figure 3. Chaotic Forced Van der Pol Oscillator with Integral Backstepping Controller (a) Time series (b) Phase portrait (c)Total Harmonic Distortion diagram 
Van Der Pol, position vs, time, $\mathrm{c}=\mathbf{2 . 0 0}$

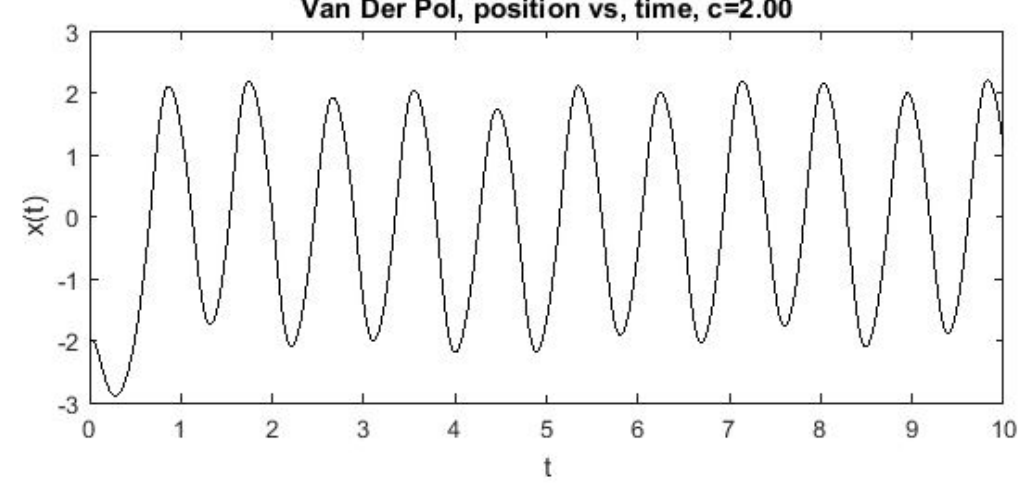

(a)

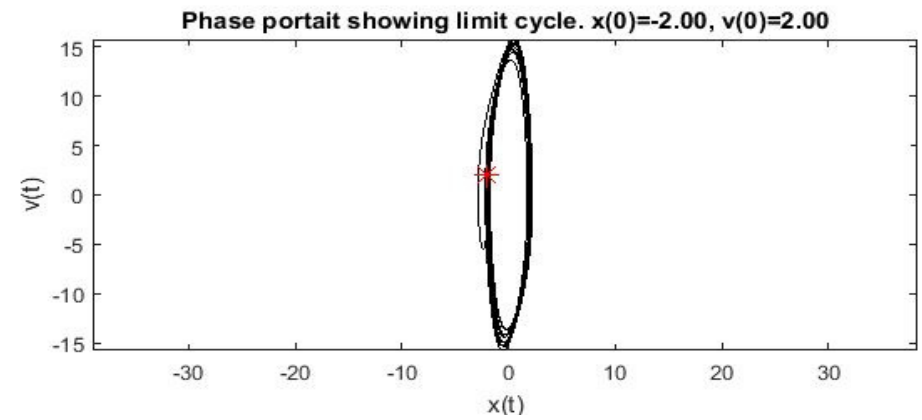

(b)

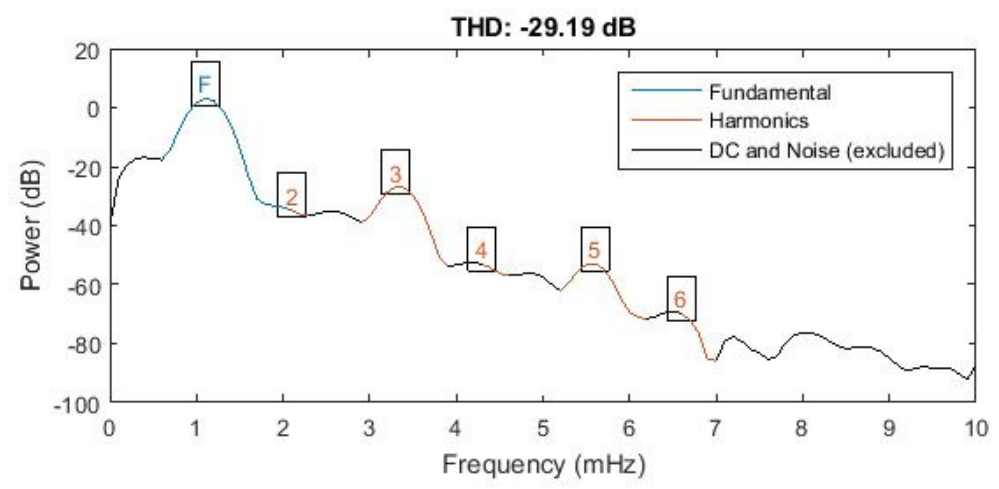

(c)

Figure 4. Chaotic Forced Van der Pol Oscillator with matched disturbance and Integral Backstepping Controller (a) Time series (b) Phase portrait (c)Total Harmonic Distortion diagram

Figure 5(a)-(c) shows the performance of integral backstepping sliding mode control method on a chaotic forced Van der Pol oscillator. The resulting signal shows a little deviation from the evolving value at position $\mathrm{x}(\mathrm{t})=-2.274$ and $\mathrm{x}(\mathrm{t})=1.936$ at $\mathrm{t}=0.232 \mathrm{sec}$ and $\mathrm{t}=0.856 \mathrm{sec}$. The resulting signal oscillates between the evolving values. $x(t)$ approaches $(-2,2)$, therefore, the dynamic of the system is bistable. The phase portrait shows a good improvement in chaotic behavior of the forced Van der Pol oscillator. The result of the simulation implies that chaos has 
been eliminated to a large extent as shown in the phase portrait compared to forced Van der Pol oscillator without a control signal applied. The integral backstepping sliding mode control of chaotic forced Van der Pol oscillator is seen to have a THD value of $-16.65 \mathrm{~dB}$ without matched disturbance which this implies that the signal emission has a $14.7 \%$ distortion.

Figure 6(a)-(c) show the performance of integral backstepping sliding mode control method on a chaotic forced Van der Pol oscillator with matched disturbance. The resulting signal shows a little deviation from the evolving values at position $\mathrm{x}(\mathrm{t})=-2.27$ and $\mathrm{x}(\mathrm{t})=2.02$ and $\mathrm{t}=0.22 \mathrm{sec}$ and $\mathrm{t}=0.85 \mathrm{sec}$. It quickly approaches stable oscillation between evolving values. The result of the simulation implies that integral backstepping sliding mode control method has been able to eliminate chaos to a large extent as shown in the time plot and phase diagram. The integral backstepping sliding mode control of chaotic forced Van der Pol oscillator is seen to have a THD value of $-16.85 \mathrm{~dB}$ with matched disturbance. This implies that the signal emission has a $14.4 \%$ distortion.

The integral backstepping sliding mode control method was simulated at damping function $\mathrm{c}=2$, amplitude $\mathrm{p}=100$ and angular frequency $\mathrm{w}=7$. The phase portrait shows an improvement in chaos control as compared to the forced Van der Pol oscillator without a control signal applied. The control method has thus been able to drive the oscillator to stability and is also robust towards a matched disturbance.

\section{Conclusions}

This research work was successful in addressing the problem of stability and chaos control in a forced Van der Pol oscillator. It has also showed robustness towards matched and unmatched disturbance. MATLAB/M-FILE was used to simulate Integral Backstepping Sliding Mode Control of a chaotic forced Van der Pol oscillator. The results showed that Integral Backstepping Sliding Mode Control method was able to reach stability in a short time and it maintained oscillation between the evolving values. The sliding surface parameter $\gamma 1$ and the sign function played significant part in ensuring the stability of this control method. It can also be seen that Integral Backstepping Sliding Mode Control method has a 14.7\% Total Harmonic Distortion however, Passive filters, Shunt passive filters, Series passive filters, Low pass filters, or Active filters can be applied to this control method to control the Harmonic Distortion.

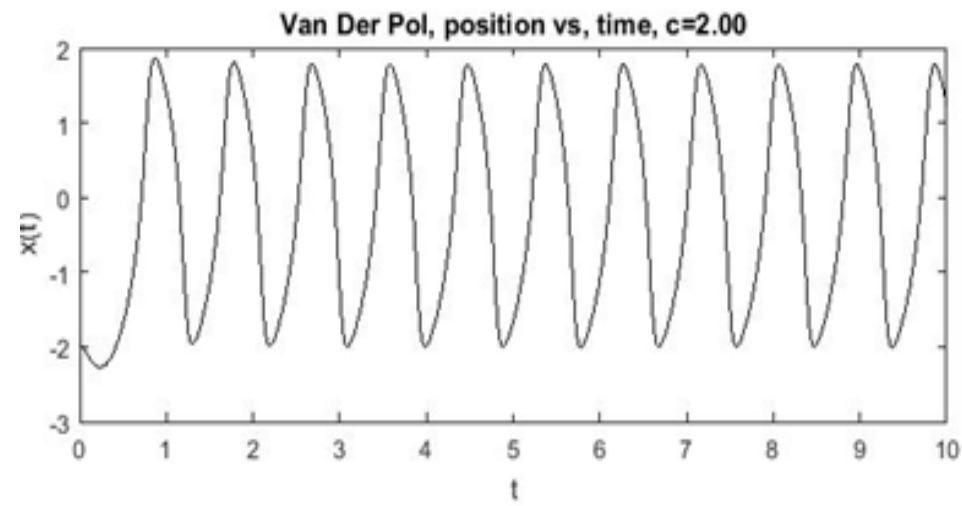

(a) 
International Journal of Control Theory and Computer Modeling (IJCTCM) Vol.8, No.1/2/3/4, October 2018

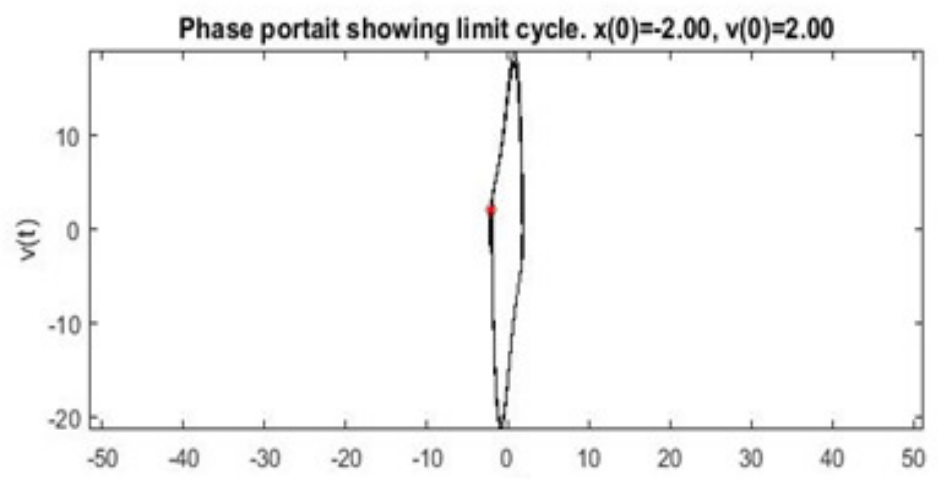

(b)

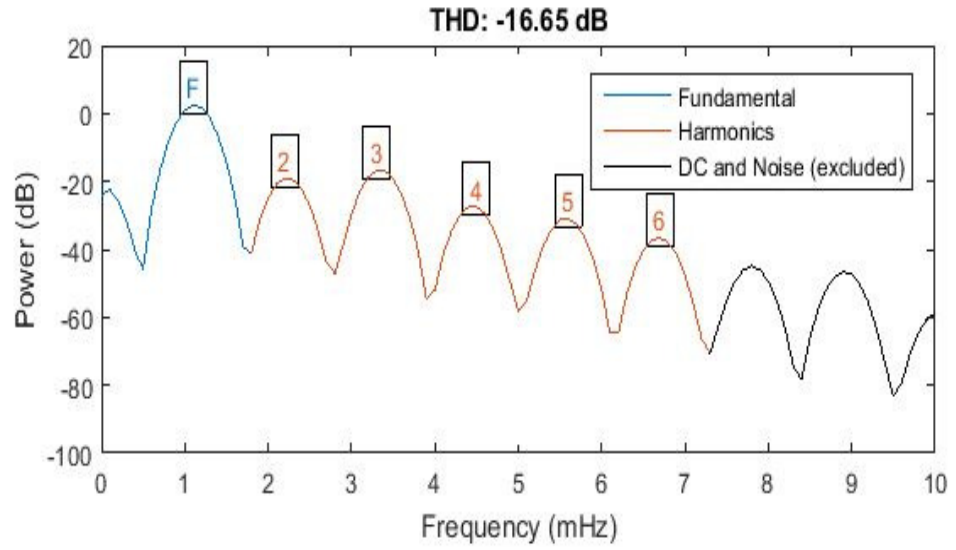

(c)

Figure 5. Chaotic Forced Van der Pol Oscillator with Integral Backstepping Sliding Mode Controller (a) Time series (b) Phase portrait (c)Total Harmonic Distortion diagram

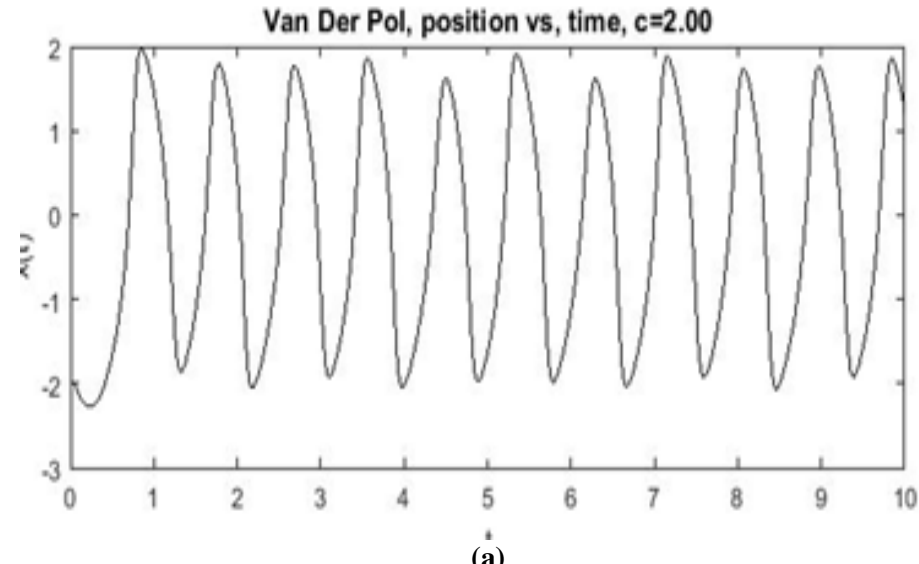




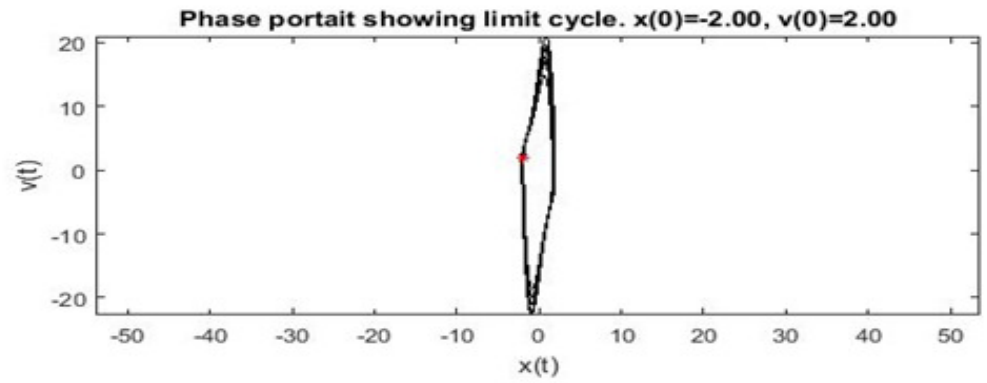

(b)

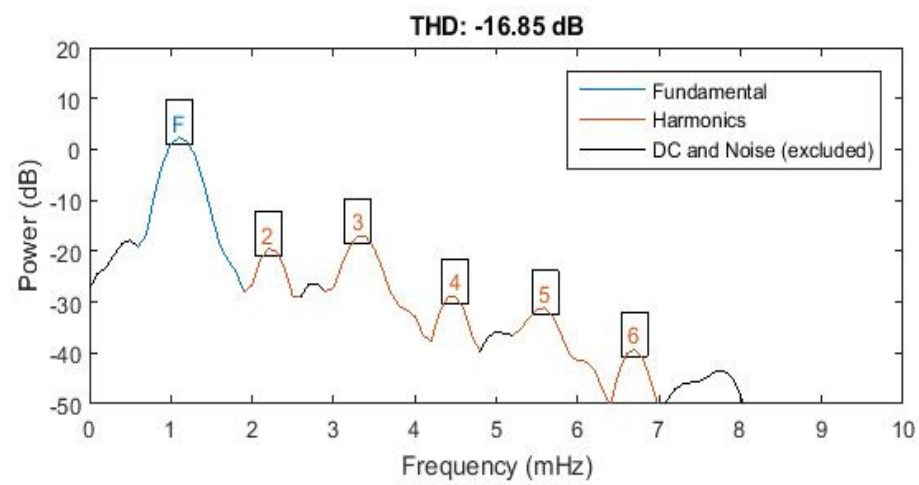

(c)

Figure 6. Chaotic Forced Van der Pol Oscillator with matched disturbance and Integral Backstepping Sliding Mode Control (a) Time series (b) Phase portrait (c)Total Harmonic Distortion diagram

\section{REFERENCES}

[1] R. S. Barbosa, J. A. T. Machado, B. M. Vinagre, and A. J. Calderon, (2007). "Analysis of the Van der Pol oscillator containing derivatives of fractional order," J. Vib. Control, Vol. 13, No. 9-10, pp. 12911301.

[2 H. K. Khalil, (2000). “Nonlinear Systems”.Prentice Hall, pp. $41-47$.

[3] A. Nabanita, and M. Chitralekha, (2013). "Integral backstepping sliding mode control for underactuated systems: Swing-up and stabilization of the Cart-Pendulum System”. ISA Transactions. India, pp 870-880.

[4] J. J. Slotine and W. Li, (1991). “Applied Nonlinear Control”. Prentice-Hall. London.

[5] A. Fradkov, A. Pogromsky, and A. Yu, (1996). "Introduction to Control of Oscillations and Chaos". World Scientific, Singapore.

[6] E. Ott, C. Grebogi, and J.A. Yorke, (1990). "Controlling chaos". Physical Review Letters, vol. 64, pp 1196-1200.

[7] J. Lu, and S. Zhang, (2001). “Controlling Chen's chaotic attractor using backstepping design based on parameters identification". Phys Lett A, vol. 256, pp148-152.

[8] Y. Zeng, S. N. Singh, (1997). "Adaptative control of chaos in Lorenz system”, Dynamic and Control, vol. 7, pp 143-154. 
[9] H. Kotaro, W. Xiaofeng, M. Junichi, H. Jinglu, and Chunzhi Jin, 2000. "Universal learning network and its application to chaos control”. Elsevier Science, vol. 13, pp. 239-253

[10] E.N. Sanchez, J.P. Perez, M. Martinez, and G. Chen, (2002). "Chaos stabilization: an inverse optimal control approach.” Latin Amer Appl Res Int J., vol. 32, pp 111-114.

[11]S. Ge, C. Wang, and T.H. Lee, (2000). "Backstepping control of a class of chaotic systems". International Journal of Bifurcation and Chaos, vol. 10, pp 1149-1162.

\section{Authors}

Ismaila Adeniyi Kamil obtained a B.Sc. Degree in Electronic \& Electrical Engineering from Obafemi Awolowo University, Ile-Ife, Nigeria in 1989, M.Phil. Degree in Electrical Engineering from University of Lagos, Nigeria in 1997 and a Ph.D. in Communication Engineering from University of Ibadan, Nigeria in 2010. He is currently a Senior Lecturer in the Department of Electrical and Electronic Engineering, University of Ibadan, Nigeria. His areas of interest include Signal Processing, Wireless Communication and Control \& Instrumentation.

Samuel Oyelekan Oladipo obtained a B.Tech. Degree in Electronic \& Electrical Engineering from Ladoke Akintola University of Technology Ogbomoso Nigeria in 2014 and M.Sc. Degree in Electronic \& Electrical Engineering from University of Ibadan, Nigeria in 2018. His areas of interest include Nonlinear control, Chaos Control and Stability.
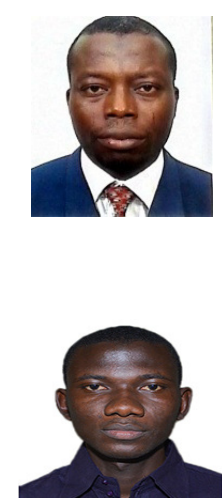